Original paper

\title{
The effect of water intake on ultrasound tissue characteristics and hemodynamics of adult livers
}

\author{
Jason Lee' ${ }^{1}$, Rebecca Lee', Todd Erpelding², Roger L. Siddoway³ ${ }^{2}$ Jing Gao ${ }^{1}$ \\ 'Rocky Vista University, USA \\ ${ }^{2}$ Canon Medical Systems, USA \\ ${ }^{3}$ Utah Gastroenterology, USA
}

\begin{abstract}
Aim of the study: To assess the effect of water intake on ultrasound tissue characteristics and hemodynamics of adult livers.

Material and methods: In February 2020, we prospectively performed ultrasound shear wave elastography and attenuation imaging (ATI) of the liver parenchyma, and spectral Doppler sonography of the portal vein and hepatic artery in 19 adult healthy volunteers ( 10 men and 9 women, mean age 27 years, mean body mass index $24.65 \mathrm{~kg} / \mathrm{m}^{2}$ ). We measured liver shear wave velocity $(\mathrm{SWV}, \mathrm{m} / \mathrm{s})$, shear wave dispersion $(\mathrm{SWD}, \mathrm{m} / \mathrm{s} / \mathrm{kHz})$, attenuation coefficient $(\mathrm{dB} / \mathrm{cm} / \mathrm{MHz})$, main portal vein velocity (PVV, $\mathrm{cm} / \mathrm{s})$, hepatic artery peak systolic velocity (PSV, $\mathrm{cm} / \mathrm{s})$, and end diastolic velocity $(E D V, c m / s)$ immediately before and at different time points $(15,30,45$, and 60 minutes) after water intake (1.0 I water and $1.5 \mathrm{I}$ water for body weight $<150 \mathrm{lbs}$. and $\geqslant 150 \mathrm{lbs}$., respectively).

Results: The differences in SWV, PVV, hepatic artery PSV and EDV before and after water intake were significant $(p<0.01)$ whereas the differences in SWD and ATI were not $(p>0.05)$ based on repeated measures ANOVA tests. The values of SWV, PVV, PSV, and EDV reached a peak at 30-45 minutes and returned to baseline 60 minutes after water intake. We observed positive correlations of SWV with PVV, PSV, and EDV in linear regression analyses $\left(r^{2}>0.73\right)$

Conclusions: Water intake affects the liver stiffness and hemodynamics. No water intake at least one hour prior to liver ultrasound elastography and Doppler sonography is recommended.
\end{abstract}

Key words: Doppler sonography, liver, shear wave elastography, ultrasound.

Address for correspondence:

Dr. Jing Gao, Rocky Vista University, United States, e-mail: jgao@rvu.edu

\section{Introduction}

Chronic liver disease with variable etiologies may lead to liver cirrhosis, a prominent cause of mortality in many developed and developing countries [1]. Early detection of chronic liver disease is challenging. Liver biopsy was considered the gold standard, but due to its invasive nature, risk of serious complications, as well as sampling error, quantitative ultrasound imaging techniques including ultrasound elastography are becoming more frequently used, with the potential for replacing liver biopsy in the assessment of some chronic diffuse liver diseases in combination with liver biochemical markers [2-5]. Ultrasound elastography of the liver has proven to have good sensitivity and specificity for evaluating liver fibrosis in chronic liver diseases $[6,7]$.

There are various pathologic changes that can lead to an alteration in the tissue stiffness, as well as at the microstructural level of liver tissue [8]. Liver stiffness has been shown to be a reliable indicator of the degree of liver fibrosis and can also predict progression of diseases such as non-alcoholic fatty liver disease (NAFLD), hepatocellular carcinoma (HCC), viral hepatitis, and liver congestion [5-11]. Besides liver diseases, physiologic conditions (hydration, food intake) can also induce a change in the liver stiffness [12-14]. Changes in the liver tissue can be assessed by ultrasound imaging techniques, including ultrasound shear 
wave velocity (SWV), shear wave dispersion (SWD), and attenuation imaging (ATI) [3, 7, 15-18], among others. In SWV, shear waves are produced by pushes of acoustic radiation force impulse. Unlike longitudinal ultrasound waves, the shear wave is a transverse wave that travels perpendicularly to the emitting ultrasound beam from the transducer and propagates at a slower velocity $[5,7]$. The degree of liver stiffness can be determined by the speed of the shear wave propagation in the tissue [2-5]. The stiffness of the tissue can be expressed by either SWV $(\mathrm{m} / \mathrm{s})$ or Young's modulus $(\mathrm{kPa})$. A high value of SWV represents a stiff tissue whereas a low value of SWV represents a soft tissue [2,3]. SWD $(\mathrm{m} / \mathrm{s} / \mathrm{kHz})$, on the other hand, assesses the dispersion slope of the shear wave speed that is dependent on shear wave frequency $[15,16]$. SWD is primarily related to the viscosity of the liver and has been shown to be a more accurate measurement of liver inflammation than SWV [9]. ATI $(\mathrm{dB} / \mathrm{cm} / \mathrm{MHz})$ measures the attenuation coefficient caused by diffusion, reflection, absorption, and scattering of the ultrasound beam in the tissue $[8,17]$. A positive correlation has been found between increasing ATI value and high grade of liver steatosis, and it has proved to be a great screening tool for patients with hepatic steatosis $[17,18]$.

Conventional 2D Doppler sonography has been used to assess liver hemodynamics [19]. Two vessels supply blood flow to the liver. Doppler velocity of the portal vein has been used to monitor the change in liver hemodynamics in liver disease (liver cirrhosis) and physiologic condition (water intake) [20-22]. We have not found an article discussing the correlation between the change of ultrasound tissue characteristics (SWV, SWD, ATI) and the change in hemodynamics of the hepatic artery after water intake in normal human liver during our literature review.

The aim of this study was to determine whether water intake alters measurement of liver elasticity, viscosity, attenuation, and hemodynamics and provide further evidence supporting the common practice of fasting prior to liver ultrasound examination.

\section{Material and methods}

\section{Subjects}

The Institutional Review Board approved the study (IRB\# 2019-0009). All participants provided written informed consent before ultrasound scanning.

In February 2020, we consecutively recruited 19 healthy volunteers ( 10 males and 9 females, mean age 27 years, age range 24-38 years, mean body mass index $=24.65 \mathrm{~kg} / \mathrm{m}^{2}$ ). Inclusion criteria for recruiting adult healthy volunteers in the study included age of 20 years or older; no history of liver or biliary disease of any kind; no medication that may affect liver function for three months; no history of surgery, trauma, or interventional procedure in the liver; tolerant to ultrasound examination and drinking water; being able to sign written informed consent.

\section{Ultrasound data acquisition}

An Aplio i800 ultrasound scanner (Canon Medical Systems USA, Tustin, CA, USA) equipped with a curvilinear probe (PVI-475 BX, 1.6-6.2 MHz) was used to acquire all ultrasound measurements. Manufacturer-recommended machine settings for liver ultrasound (pre-settings for grayscale image, shear wave elastography, ATI, and spectral Doppler) were used to acquire all ultrasound parameter measurements in all subjects.

Subjects were asked to lie in the left lateral recumbent position with their right arm behind their head. Subjects were draped with disposable paper drapes during scanning for privacy. An appropriate amount of gel was applied to the region of interest and the ultrasound probe was placed in the right upper quadrant of the subject's abdomen, on the midclavicular, or anterior axillary line between the intercostal spaces. Subjects were asked to hold their breath during active data collection to minimize excessive motion-induced image artifacts and variation in Doppler sonography and shear wave elastography [11, 18, 22].

Each subject was scanned a total of 5 times in increments of 15 minutes. Each round of scans spanned 5-7 minutes. The first scan was taken after 4-6 hours of nothing to eat/drink (NPO) in order to establish a baseline. Subjects were then asked to consume a predetermined amount of water based on the subject's body weight (1.0 l of water for < $150 \mathrm{lbs}$., $1.5 \mathrm{l}$ of water for $\geq 150 \mathrm{lbs}$.) within 3 to 5 minutes. The protocol was adopted from a previous study investigating the changes of liver stiffness after ingestion of $1.5 \mathrm{l}$ of water in 9 males and 1 female [14]. The remaining four scans were subsequently taken in 15-minute increments after water intake (15, 30, 45, and 60 minutes after drinking water).

Each round of scans included the following measurements: 6 of SWV, 6 of SWD (Fig. 1), 5 of ATI (Fig. 2), 2 of Doppler for flow velocity of the portal vein, and 2 of the common hepatic artery (Fig. 3). The average of each ultrasound parameter's measurements at each time point was used for analysis. A total of 5 rounds of scan (one immediately before drinking water and four after drinking water in 15-minute increments) were taken for each subject.

A senior operator (J.G.) with 30 years' experience in abdominal ultrasound and 10 years in ultra- 

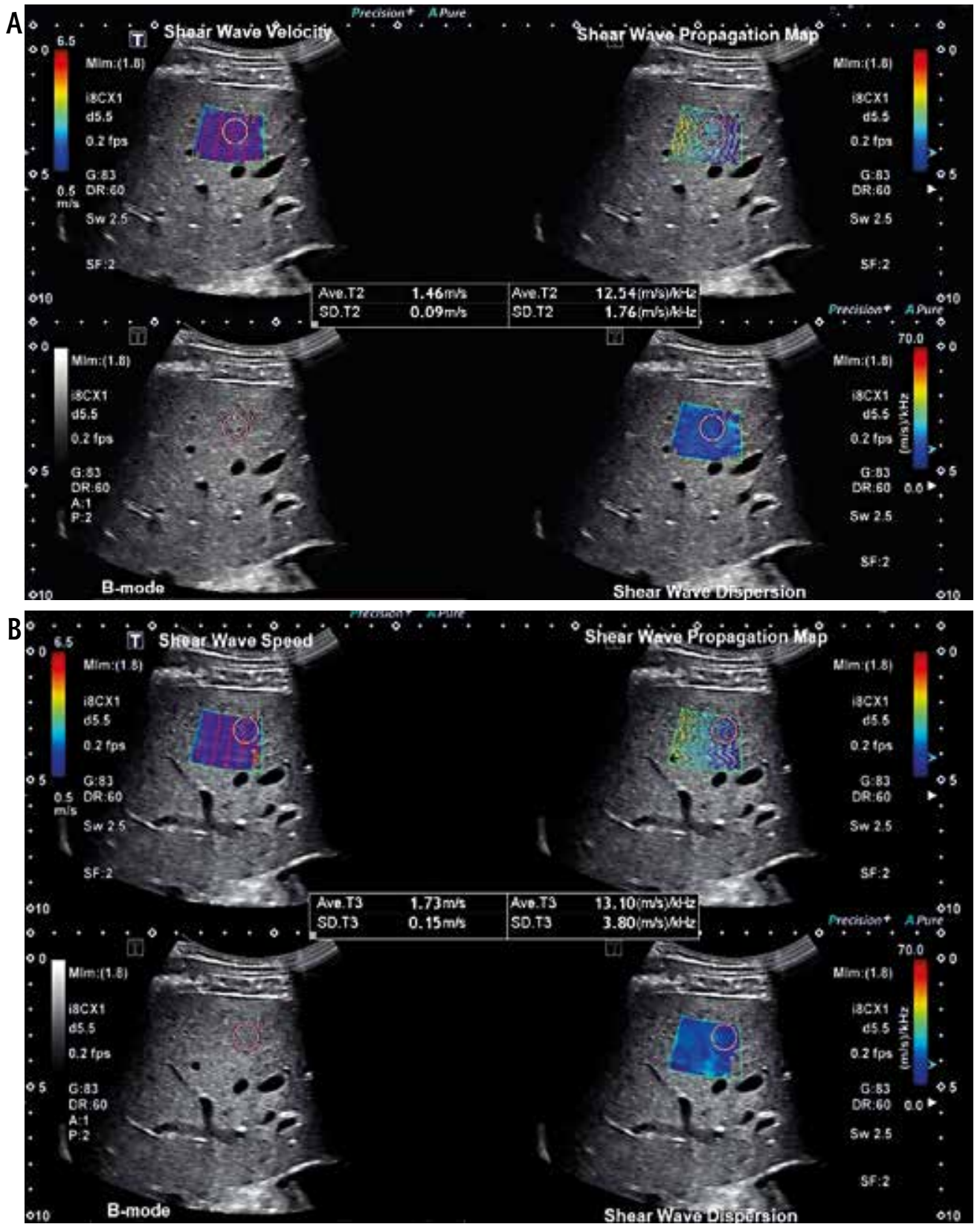

Fig. 1. Ultrasound shear wave velocity (SWV, $\mathrm{m} / \mathrm{s}$ ) and shear wave dispersion $(\mathrm{SWD}, \mathrm{m} / \mathrm{s} / \mathrm{kHz}$ ) are measured immediately before $(\mathbf{A})$ and 45 minutes (B) after water intake. SWV, shear wave speed map, grayscale image, and SWD are displayed in QuadView image. The increase of SWV after water intake is significant $(1.46 \mathrm{~m} / \mathrm{s}$ vs $1.73 \mathrm{~m} / \mathrm{s})$ whereas SWD is not $(12.54 \mathrm{~m} / \mathrm{s} / \mathrm{kHz}$ vs. $13.10 \mathrm{~m} / \mathrm{s} / \mathrm{kHz})$

sound elastography and a junior operator (R.L.) with two years' training in abdominal ultrasound and two weeks' training in ultrasound elastography performed ultrasound scans in 19 subjects.

\section{Statistical analysis}

All variables of ultrasound parameters at different time points are expressed as mean and standard deviation. The data were compiled and organized by variable and time increment obtained. ANOVA with repeated measures was performed for statistical analysis in order to determine the difference of each variable among the different time points. A $t$-test was used to analyze the differences in ultrasound parameters between paired groups. A p-value was assigned to compare the null hypothesis against the alternative hypothesis with a $p$-value $<0.05$ indicating a significant difference between the two hypotheses. Linear regression was also performed comparing each variable measuring tissue characteristic changes in the liver parenchyma (SWV, SWD, and ATI) to each variable measuring hemodynamic changes in the liver vessels [portal vein velocity (PVV), he- 

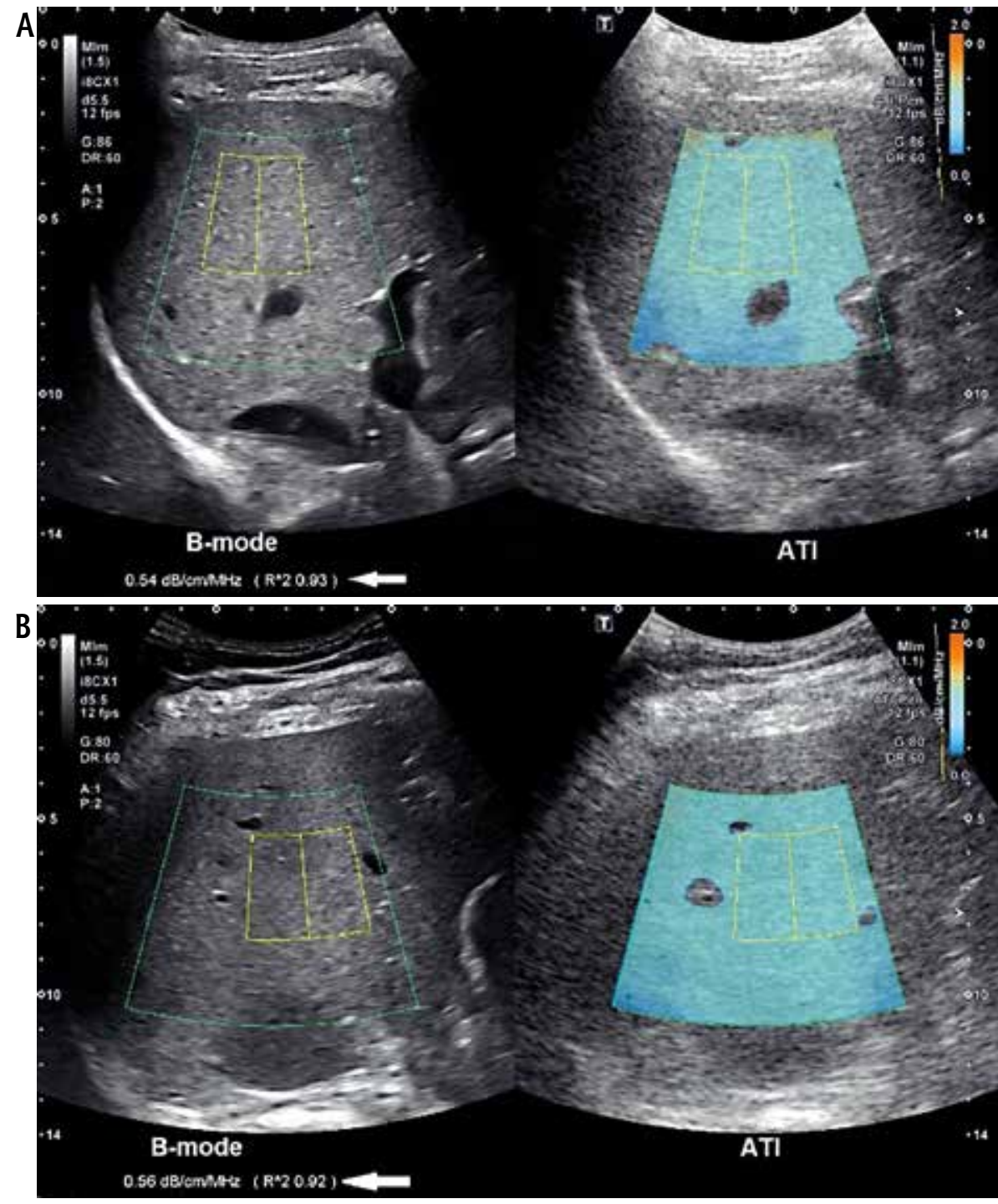

Fig. 2. Ultrasound attenuation imaging was performed before $(\mathbf{A})$ and after $(\mathbf{B})$ water intake. There is no significant difference in attenuation coefficient in the liver parenchyma after drinking water $(0.54 \mathrm{~dB} / \mathrm{cm} / \mathrm{MHz}$ vs. $0.56 \mathrm{~dB} / \mathrm{cm} / \mathrm{MHz})$

patic artery peak systolic velocity (PSV) and end diastolic velocity (EDV)]. A strong positive correlation between variables was suggested if $r^{2} \geq 0.7$ [24]. Statistical analysis was performed with commercially available software (SPSS Statistics, Version 27, IBM).

\section{Results}

Ultrasound parameters were successfully measured in all 19 subjects. No subjects were excluded from the statistical analysis.

The mean and standard deviation of all measured variables, evaluating both liver parenchyma tissue characteristics (SWV, SWD, ATI) and liver hemodynamics (PVV, hepatic artery PSV and EDV) before and at four time points after drinking water, are presented in Fig. 4. Results of the linear regression are listed in Table 1.
We observed an increase in values of ultrasound parameters over the five different time increments. Increases of SWV, PVV, and hepatic artery PSV, EDV values were significantly different $(p<0.01)$ whereas SWD and ATI values were not significantly different $(p>0.05)$ after drinking water compared to those values before water intake. The peak values of SWV (1.53 $\pm 0.1 \mathrm{~m} / \mathrm{s}), \operatorname{PVV}(34.4 \pm 6.96 \mathrm{~cm} / \mathrm{s})$, and hepatic artery $\operatorname{EDV}(36.19 \pm 12.71 \mathrm{~cm} / \mathrm{s})$ were at 45 minutes after water intake. The peak values of SWD $(13.70 \pm 2.801 \mathrm{~m} / \mathrm{s} /$ $\mathrm{kHz})$ and hepatic artery PSV $(98.25 \pm 28.48 \mathrm{~cm} / \mathrm{s})$ were at 30 minutes after water intake. ATI value $(0.62 \pm 0.12$ $\mathrm{dB} / \mathrm{cm} / \mathrm{MHz}$ ) peaked at 15 minutes after water intake. All ultrasound parameters returned to near before water intake values by 60 minutes after water intake.

Based on the ANOVA with repeated measure analysis, of the three parameters (SWV, SWD, ATI) used to 

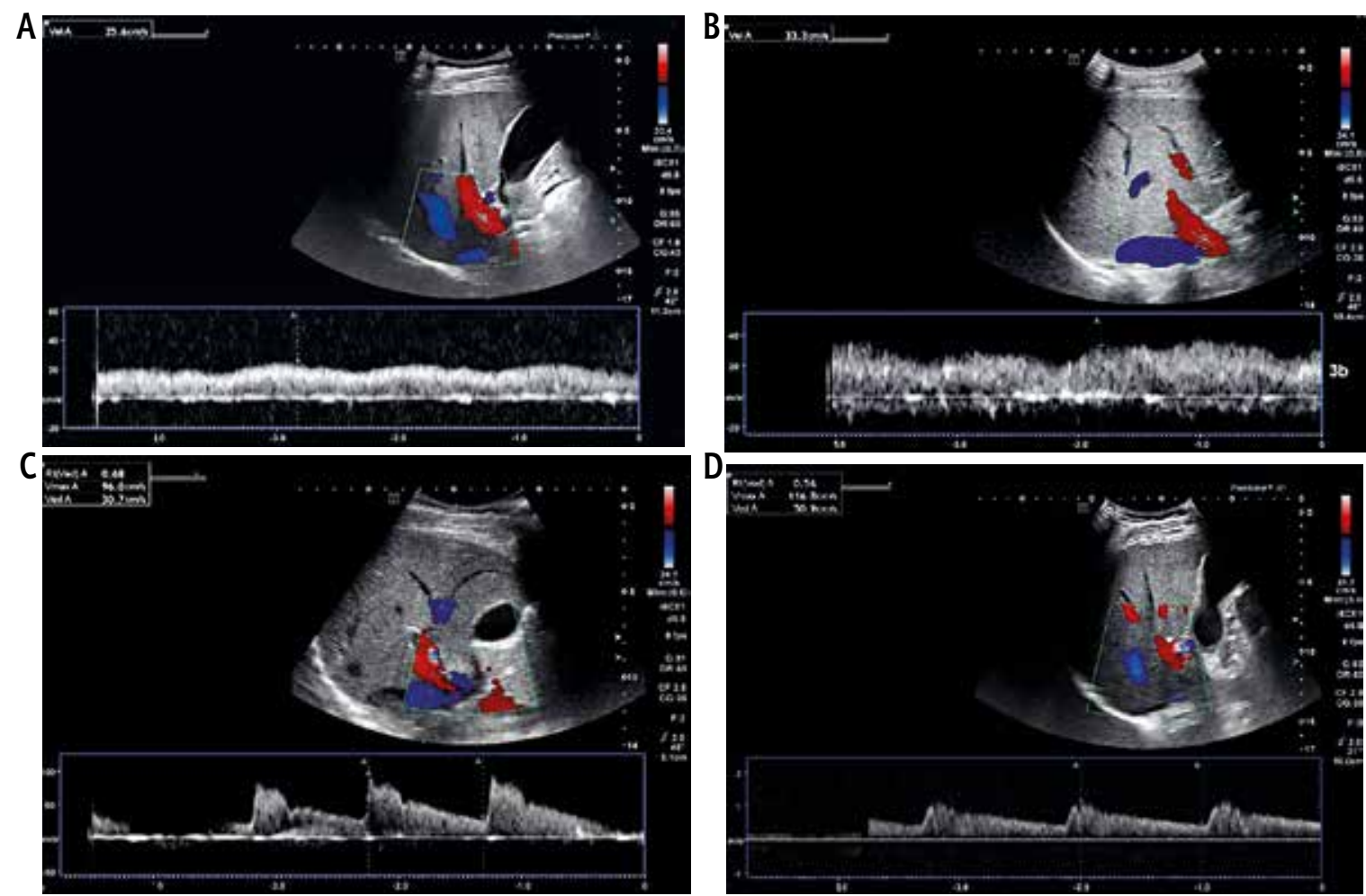

Fig. 3. Using Doppler angle correction, flow velocity in the main portal vein (PVV) and hepatic artery are measured immediately before and at different time points after water intake. Portal vein velocity measures $25.6 \mathrm{~cm} / \mathrm{s}$ before $(\mathbf{A})$ and $33.3 \mathrm{~cm} / \mathrm{s}$ at 45 minutes after $(\mathbf{B})$ water intake. Hepatic artery peak systolic velocity (PSV) and end diastolic velocity (EDV) measure $96.0 \mathrm{~cm} / \mathrm{s}$ and $30.7 \mathrm{~cm} / \mathrm{s}$ before (C) and $116.8 \mathrm{~cm} / \mathrm{s}$ and $50.9 \mathrm{~cm} / \mathrm{s}$ after (D) water intake. There are clear increases in PVV, PSV and EDV after water intake $(p<0.01)$. Vel A - velocity (A and B), Vmax A - artery peak systolic velocity, Ved A - artery end diastolic velocity (C and D)

measure liver tissue characteristics, only SWV demonstrated a significant difference in measured values across five different time increments $(p<0.001)$. No significant differences in measured values of SWD and ATI over time were found (all $p>0.05$ ). Interestingly, all the measured variables for evaluating the change in hemodynamics in the liver (PVV, PSV, and EDV) demonstrated statistical significance $(p<0.01)$.

Results from the linear regression demonstrated a strong positive correlation of SWV with PVV $\left(r^{2}=0.932\right)$, PSV $\left(r^{2}=0.796\right)$, and $\operatorname{EDV}\left(r^{2}=0.726\right)$. The correlation between SWD and PSV was positive $\left(r^{2}=0.557\right)$ but not significant $(p=0.147)$. No significant correlation between SWD and PVV, between SWD and EDV, or between ATI and any of the Doppler variables measuring hemodynamic changes in the liver was observed (Table 1).

\section{Discussion}

The results demonstrate that water intake affects the hemodynamics of the liver as assessed by Doppler flow parameters and subsequently affects the liver stiffness measured with ultrasound shear wave velocity. The change in mean values of SWV significantly correlates with the changes in PVV and hepatic artery PSV, EDV at the different intervals of time. The measured values of SWV, SWD, PVV, PSV, and EDV peaked 30-45 minutes after water intake and returned to near baseline within 60 minutes after drinking water. Further analysis with ANOVA confirmed that the differences in the means across time for SWV, PVV, PSV, and EDV were significant. Analysis with linear regression provided further evidence supporting this conclusion by demonstrating a strong positive correlation of SWV with PVV, PSV, and EDV. This conclusion is consistent with the use of SWV to measure liver stiffness $[3,7,25]$. However, it is important to note that SWV is susceptible to hemodynamic changes in the liver. The increase of blood flow in the portal vein, hepatic artery, or both may be one of the important factors in altering the liver stiffness. Our findings support the viability of the common practice of no water intake prior to ultrasound evaluation of the liver in order to obtain more accurate measurements of liver stiffness. A standard procedure, especially with patient preparation, is needed to obtain reliable values and avoid overestimation of liver stiffness.

Although a strong positive correlation was found between SWV and hemodynamic changes in the liver, 

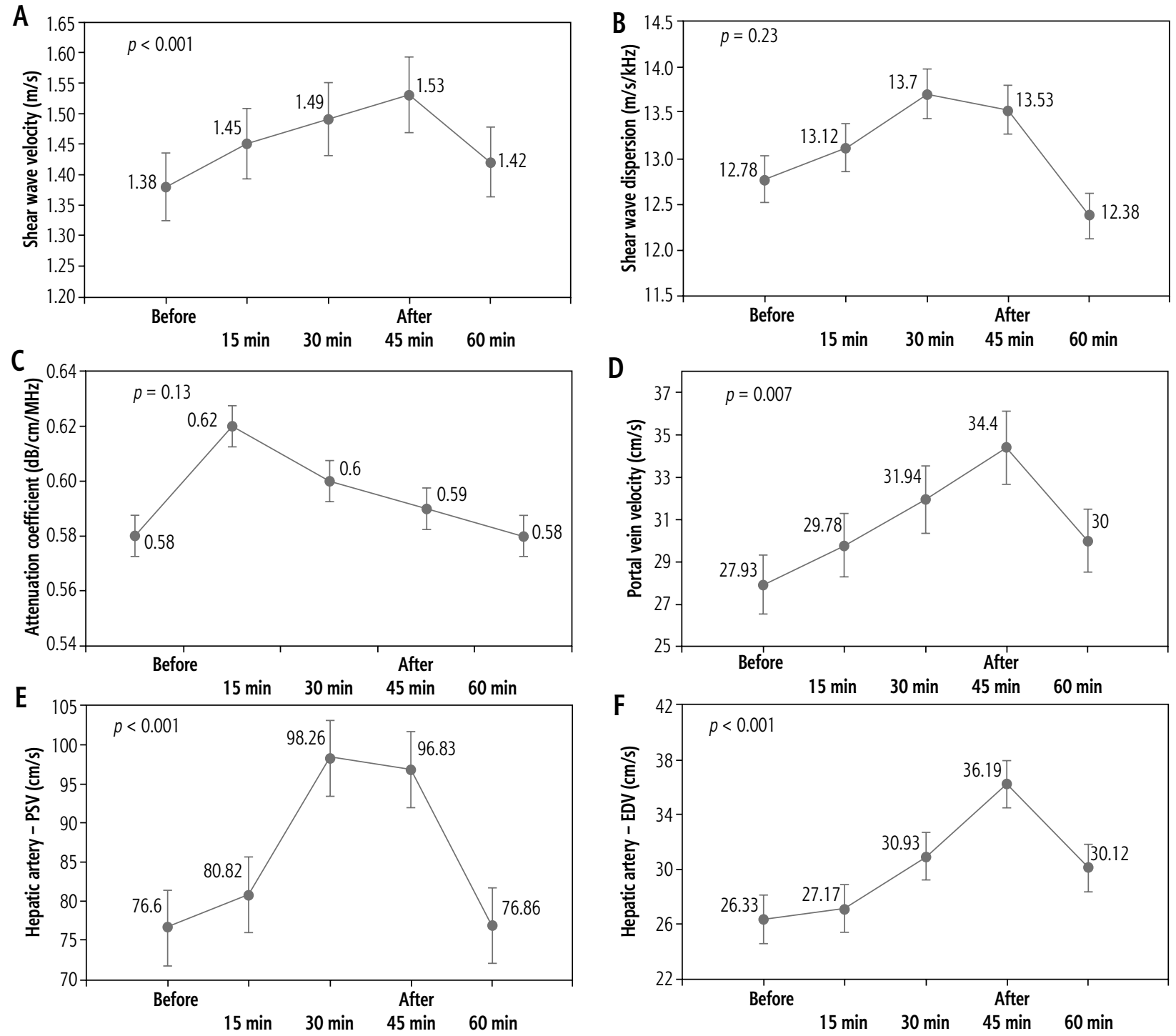

Fig. 4. Line plots show the mean value and standard deviation of shear wave velocity (SWV, A), shear wave dispersion (SWD, B), attenuation coefficient (C), portal vein velocity (PVV, D), hepatic artery peak systolic velocity (PSV, E), and hepatic artery end diastolic velocity (EDV, F) measured immediately before and 15, 30, 45, and 60 minutes (min) after water intake. Based on ANOVA with repeated measures analyses, differences in SWV, PVV, hepatic artery PSV and EDV at different time points were significant $(p<0.01)$, but the difference in SWD or ATI at different time points was not significant $(p>0.05)$

Table 1. Correlations between ultrasound tissue characteristics and hemodynamic parameters

\begin{tabular}{lccc}
\hline Outcome measures $\left(r^{2} / p\right)^{*}$ & SWV $(\mathrm{m} / \mathrm{s})$ & SWD $(\mathrm{m} / \mathrm{s} / \mathrm{kHz})$ & ATI $(\mathrm{dB} / \mathrm{cm} / \mathrm{MHz})$ \\
\hline Portal vein velocity $(\mathrm{cm} / \mathrm{s})$ & $0.932 / 0.008$ & $0.48 / 0.195$ & $0.043 / 0.739$ \\
\hline Hepatic artery PSV $(\mathrm{cm} / \mathrm{s})$ & $0.796 / 0.042$ & $0.557 / 0.147$ & $0.027 / 0.792$ \\
\hline Hepatic artery EDV $(\mathrm{cm} / \mathrm{s})$ & $0.726 / 0.047$ & $0.289 / 0.391$ & $0.003 / 0.933$ \\
\hline
\end{tabular}

$\left(r^{2} / p\right)^{*}$ - the values of $r^{2}$ are coefficients of determination based on linear regression analyses and $p$ values for the coefficients indicate whether these relationships are statistically significant; ATI - attenuation imaging, EDV - end diastolic velocity, PSV - peak systolic velocity, SWD - shear wave dispersion, SWV - shear wave velocity

this was not the case for SWD or ATI. Much like SWV, the mean values of SWD and ATI peaked 15-30 minutes following water intake and returned to near baseline within 60 minutes; however, further analysis with ANOVA with repeated measures did not detect statis- tically significant differences between the measured values at different time intervals. Linear regression showed weak and/or non-significant correlations of SWD and ATI with PVV, PSV, and EDV. While SWD and ATI are often used in conjunction with SWV to 
assess pathologic changes in the liver, SWD is known to be a better measure of tissue viscosity $[9,16]$, and ATI is known as a better measure of steatosis $[17,18]$. In the study, we did not observe significant alterations of SWD or ATI following hemodynamic changes induced by water intake in the normal young adult liver compared to SWV. The results did not show significant effects of water intake on SWD and ATI of the liver.

There were some limitations to our study. First, the number of subjects enrolled in the study was small, and they were primarily young, healthy individuals. In a normal healthy liver, the change in liver stiffness as a result of water ingestion may not be as significant or pronounced as compared to individuals with evidence of liver with pathologic changes (such as fibrosis). We do not know whether changes in ultrasound parameters occurring as the effects of water intake on liver tissue characteristics and hemodynamics could be observed in patients with liver diseases, or the results may vary. Second, we did not have liver histology as a reference standard to correlate with the changes in ultrasound parameters of the liver, although liver biopsy is not the standard of care when there are no clinical indications of liver diseases. Third, we did not observe stronger correlations of SWD and ATI with PVV, PSV, or EDV in normal young adult livers; the results may differ in patients with liver diseases, such as liver fibrosis and/or steatosis. Fourth, our study only evaluated changes in the liver up to an hour following water intake. We did not extend the time of observation up to 2 or 3 hours after water intake. Although most measured values returned to near baseline (before drinking water) values within the given time frame, future studies could continue to evaluate changes in the measured liver ultrasound parameters. Finally, we did not test intra- and interobserver reliability in performing shear wave elastography, ATI, or Doppler sonography; however, good intra- and interobserver reliabilities in measuring liver SWV, SWD, ATI, and PVV have been reported $[17-19,25,26]$. Further study on testing the water intake effect on liver tissue characteristics and hemodynamics in a large group of subjects with and without liver diseases is warranted.

\section{Conclusions}

Water intake has significant effects on the liver shear wave velocity, portal vein velocity, and hepatic artery PSV and EDV. Fasting prior to liver shear wave elastography and Doppler sonography is highly recommended for accurate assessment of liver stiffness and hemodynamics.

\section{Acknowledgment}

We acknowledge Canon Medical Systems USA for loaning the ultrasound equipment used in this study.

\section{Disclosure}

The authors declare no conflict of interest.

\section{References}

1. Udompap P, Kim DH, Kim WR. Current and future burden of chronic nonmalignant liver disease. Clin Gastroenterol Hepatol 2015; 13: 2031-2041.

2. Mueller S, Sandrin L. Liver stiffness: a novel parameter for the diagnosis of liver disease. Hepat Med 2010; 2: 49-67.

3. Gharibvand MM, Asare M, Motamedfar A, et al. Ultrasound shear wave elastography and liver biopsy to determine liver fibrosis in adult patients. J Family Med Prim Care 2020; 9: 943-949.

4. Yan Y, Xing X, Lu Q, et al. Assessment of biopsy proven liver fibrosis by two-dimensional shear wave elastography in patients with primary biliary cholangitis. Dig Liver Dis 2020; 52: 555560.

5. Osman AM, Shimy AE, Mohamed MAEA. 2D shear wave elastography (SWE) performance versus vibration-controlled transient elastography (VCTE/fibroscan) in the assessment of liver stiffness in chronic hepatitis. Insights Imaging 2020; 11: 38.

6. Wang J, Li J, Zhou Q, et al. Liver stiffness measurement predicted liver-related events and all-cause mortality: a systematic review and nonlinear dose-response meta-analysis. Hepatol Commun 2018; 2: 467-476.

7. Lin Y, Li H, Lin C, et al. The diagnostic accuracy of liver fibrosis in non-viral liver diseases using acoustic radiation force impulse elastoraphy: a systemic review and meta-analysis. PLoS One 2020; 15: e0227358.

8. Yang KC, Liao Y, Tsui P, Yeh CK. Ultrasound imaging in nonalcoholic liver disease: current applications and future developments. Quant Imaging Med Surg 2019; 9: 546-551.

9. Sugimoto K, Moriyasu F, Oshiro H, et al. Clinical utilization of shear wave dispersion imaging in diffuse liver disease. Ultrasonography 2020; 39: 3-10.

10. Pirmoazen AM, Khurana A, El Kaffas A, Kamaya A. Quantitative ultrasound approaches for diagnosis and monitoring hepatic steatosis in nonalcoholic fatty liver disease. Theranostics 2020; 10: 4277-4289.

11. Kutty SS, Peng Q, Danford DA, et al. Increased hepatic stiffness as consequence of high hepatic afterload in the Fontan circulation: a vascular Doppler and elastography study. Hepatology 2014; 59: 251-260.

12. Petzold G, Porsche M, Ellenrieder V, et al. Impact of food intake on liver stiffness determined by $2-\mathrm{D}$ shear wave elastography: prospective interventional study in 100 healthy patients. Ultrasound Med Biol 2019; 45: 402-410.

13. Berzigotti A, De Gottardi A, Vukotic R, et al. Effect of meal ingestion on liver stiffness in patients with cirrhosis and portal hypertension. PLoS One 2013; 8: e58742.

14. Ipek-Ugay S, Tzschätzsch H, Hudert $\mathrm{C}$, et al. Time harmonic elastography reveals sensitivity of liver stiffness to water ingestion. Ultrasound Med Biol 2016; 42: 1289-1294. 
15. Urban MW, Chen S, Fatemi M. A review of shearwave dispersion ultrasound vibrometry (SDUV) and its applications. Curr Med Imaging Rev 2012; 8: 27-36.

16. Zelesco M, Welman CJ, Abbott S. Preliminary clinical experience with shear wave dispersion (SWD) imaging of the liver Ultrasound Med Biol 2019; 45: S25.

17. Tada T, Iijima H, Kobayashi N, et al. Usefulness of attenuation imaging with an ultrasound scanner for the evaluation of hepatic steatosis. Ultrasound Med Biol 2019; 45: 2679-2687.

18. Yoo J, Lee JM, Joo I, et al. Reproducibility of ultrasound attenuation imaging for the noninvasive evaluation of hepatic steatosis. Ultrasonography 2020; 39: 121-129.

19. Dietrich CF, Lee JH, Gottschalk R, et al. Hepatic and portal vein flow pattern in correlation with intrahepatic fat deposition and liver histology in patients with chronic hepatitis C. AJR Am J Roentgenol 1998; 171: 437-443.

20. Solhjoo E, Mansour-Ghanaei F, Moulaei-Langorudi R, Joukar F. Comparison of portal vein Doppler indices and hepatic vein Doppler waveform in patients with nonalcoholic fatty liver disease with healthy control. Hepat Mon 2011; 11: 7404-7414.

21. Stankovic Z, Csatari Z, Deibert P, et al. Noormal and altered three-dimensional portal venous hemodynamics in patients with liver cirrhosis. Radiology 2012; 262: 862-873.

22. Dauzat M, Lafortune M, Patriquin H, Pomier-Layrarques G. Meal induced change in hepatic and splanchnic circulation: a noninvasive Doppler study in normal humans. Eur J Appl Physiol Occup Physiol 1994; 68: 373-380.

23. Barry CT, Mills B, Hah Z, et al. Shear wave dispersion measures liver steatosis. Ultrasound Med Biol 2012; 38: 175-182.

24. Ratner B. The correlation coefficient: Its values range between +1/-1, or do they? J Target Meas Anal Mark 2009; 17: 139-142.

25. Lee DH, Cho EJ, Bae JS, et al. Accuracy of two-dimensional shear wave elastography and attenuation imaging for evaluation of patients with nonalcoholic steatohepatitis. Clin Gastroenterol Hepatol 2021; 19: 797-805.

26. Giunta M, La Mura V, Conti CB, et al. The role of spleen and liver elastography and color-Doppler ultrasound in the assessment of transjugular intrahepatic portosystemic shunt function. Ultrasound Med Biol 2020; 46: 1641-1650. 